\title{
THE MINIMAL DEGREE OF A FAITHFUL QUASI- PERMUTATION REPRESENTATION OF AN ABELIAN GROUP
}

\author{
by HOUSHANG BEHRAVESH
}

(Received 18 July, 1995)

1. Introduction. Let $G$ be a finite linear group of degree $n$; that is, a finite group of automorphisms of an $n$-dimensional complex vector space (or, equivalently, a finite group of non-singular matrices of order $n$ with complex coefficients). We shall say that $G$ is a quasi-permutation group if the trace of every element of $G$ is a non-negative rational integer. The reason for this terminology is that, if $G$ is a permutation group of degree $n$, its elements, considered as acting on the elements of a basis of an $n$-dimensional complex vector space $V$, induce automorphisms of $V$ forming a group isomorphic to $G$. The trace of the automorphism corresponding to an element $x$ of $G$ is equal to the number of letters left fixed by $x$, and so is a non-negative integer. Thus, a permutation group of degree $n$ has a representation as a quasi-permutation group of degree $n$. See [5].

By a quasi-permutation matrix we mean a square matrix over the complex field $\mathbb{C}$ with non-negative integral trace. Thus every permutation matrix over $\mathbb{C}$ is a quasipermutation matrix. For a given finite group $G$, let $p(G)$ denote the minimal degree of a faithful permutation representation of $G$ (or of a faithful representation of $G$ by permutation matrices); let $q(G)$ denote the minimal degree of a faithful representation of $G$ by quasi-permutation matrices over the rational field $\mathbb{Q}$, and let $c(G)$ be the minimal degree of a faithful representation of $G$ by complex quasi-permutation matrices. See [1].

Let $G \cong \prod_{i=1}^{r} C_{m_{i}}$ where $m_{i}$ is a prime power. As in [2], define $T(G)=\sum_{i=1}^{r} m_{i}$; when $G=1$ let $T(G)=0$. In [1] it is proved that $c(G)=q(G)=p(G)=T(G)$ if and only if $G \neq 1$ and $G$ has no direct factor of order 6 .

The quantity $p(G)$ for any abelian group depends on the decomposition of $G$ into a direct product of its cyclic subgroups [2]. In fact, if $G \neq 1$ is a finite abelian group, then $p(G)=T(G)$.

In this paper $G=\prod_{i=1}^{n} G_{i}$ will denote the direct product of the subgroups $G_{i}$ of $G$ $(1 \leq i \leq n)$.

For an abelian group $G$, the invariants $c(G)$ and $p(G)$ coincide because the Schur indices for abelian groups are trivial. We shall calculate these invariants for an arbitrary abelian group $G$. In view of [1], we need only resolve the case of an abelian group having the cyclic group $C_{6}$ as direct factor. Nevertheless our proof applies to an arbitrary finite abelian group.

The main result is that $c(G)=q(G)=T(G)-n$ for an abelian group $G$, where $n$ is the largest integer such that $C_{6}^{n}$ is a direct summand of $G$.

Lemma 1.1. Let $G$ be a finite abelian group and let $G$ be the direct product of its subgroups $L$ and $H$. Then $T(G)=T(L)+T(H)$.

Proof. See [2].

Glasgow Math. J. 39 (1997) 51-57. 
2. The minimal degree of a faithful quasi-permutation representation of an abelian group. Let $\chi$ be an irreducible character of $G$. Let $m_{Q}(\chi)$ denote the Schur index of $\chi$ in $G$ over $\mathbb{Q}$.

Lemma 2.1. Let $G$ be a finite group and let $\chi \in \operatorname{Irr}(G)$. Then $m_{\odot}(\chi) \mid \chi(1)$. Moreover when $\chi$ is linear, we have $m_{\mathrm{Q}}(\chi)=1$.

Proof. See [3, Corollary 10.2].

COROLLARY 2.2. Let $G$ be a finite group and let $m_{\mathbb{Q}}(\chi)=1$, for all $\chi \in \operatorname{Irr}(G)$. Then $c(G)=q(G)$. In particular if $G$ is a finite abelian group, then $c(G)=q(G)$.

Proof. This follows from the definitions of $c(G)$ and $q(G)$ together with Lemma 2.1.

Lemma 2.3. Let $\chi$ be a character of $G$. Then $\operatorname{ker} \chi=\operatorname{ker} \sum_{\alpha \in \Gamma(\chi)} \chi^{\alpha}$, where $\Gamma(\chi)=$ $\Gamma(\mathbb{Q}(\chi): \mathbb{Q})$. Moreover $\chi$ is faithful if and only if $\sum_{\alpha \in \Gamma(\chi)} \chi^{\alpha}$ is faithful.

Proof. It is clear that $\operatorname{ker}(\chi)=\operatorname{ker}\left(\chi^{\alpha}\right)$, for $\alpha \in \Gamma(\chi)$. However

$$
\operatorname{ker} \sum_{\alpha \in \Gamma(\chi)} \chi^{\alpha}=\bigcap_{\alpha \in \Gamma(\chi)} \operatorname{ker} \chi^{\alpha}=\operatorname{ker} \chi \text {. }
$$

Here are some well known facts about irreducible representations of finite abelian groups over $\mathbb{C}$ and $\mathbb{Q}$. See [4].

Let $G$ be a finite abelian group, let $\chi \in \operatorname{Irr}(G)$ and let $K=\operatorname{ker} \chi$. Then $G / K$ is isomorphic to a finite subgroup of $\mathbb{C}$. Therefore $G / K$ is cyclic.

Let $V$ be an irreducible $\mathbb{Q} G$-module and let $K_{1}=C_{G}(V)$ be the kernel of the representation of $G$ on $V$. Let $\xi$ be the corresponding character of $V$. Then there exists $\chi \in \operatorname{Irr}(G)$ such that $\xi=\sum_{\alpha \in \Gamma(\chi)} \chi^{\alpha}$, where $\Gamma(\chi)=\Gamma(\mathbb{Q}(\chi): \mathbb{Q})$. From Lemma 2.3 we know that $K_{1}=\operatorname{ker} \chi$, and so $G / K_{1}$ is cyclic.

As in [1, p. 303], let $A=\langle a\rangle$ be a cyclic group of order $m$. Then for each $d \mid m$, there is an irreducible $\mathbb{Q} A$-module $V(d)$ of dimension $\phi(d)$, where $\phi$ is the Euler totient function. We can take $V(d)$ to be $\mathbb{Q}\left(\xi_{d}\right)$, where $\xi_{d}$ is a primitive $d$-th root of unity, and $a$ acts on $V(d)$ as multiplication by $\xi_{d}$. Since $\sum_{d \mid m} \phi(d)=m$, the modules $V(d)$ are, up to isomorphism, all the irreducible $\mathbb{Q} A$-modules. Thus, there is exactly one for each divisor $d$ of $m$.

Lemma 2.4. Let $A=\langle a\rangle$ be cyclic of order $m$ and let $d \mid m$. Let $\chi_{d}$ denote the character of $\mathbb{Q} A$-module $V(d)$. Then $\chi_{d}(a)$ is the sum of the primitive $d$-th roots of unity, and so is equal to $\mu(d)$, where $\mu$ is the Möbius function. Proof. Let $S(d)=\chi_{d}(a)$. We have $S(1)=1$. Let $f(n)=\sum_{d \mid n} S(d)$. This is the sum of all
$n$-th roots of unity. Therefore

$$
\sum_{d \mid n} S(d)=1+\varepsilon+\ldots+\varepsilon^{n-1}=\frac{\varepsilon^{n}-1}{\varepsilon-1},
$$

where $\varepsilon$ is a primitive $n$-th root of unity. Hence

$$
\sum_{d \mid n} S(d)= \begin{cases}1, & \text { if } n=1 \\ 0, & \text { if } n>1\end{cases}
$$


Then, by the Möbius inversion formula, we have $S(n)=\sum_{d \mid n} \mu\left(\frac{n}{d}\right) f(d)=\mu(n)$ for $n \geq 1$.

Lemma 2.5. Let $A$ be cyclic of order $m$ and let $b$ be an element of $A$ of order $d \mid m$. Then $\chi_{m}(b)=\frac{\phi(m)}{\phi(d)} \mu(d)$. In particular, $\chi_{m}$ is faithful and is the only faithful character of an irreducible $\mathbb{Q}$-representation of $A$.

Proof. See [1, Lemma 3.4].

Corollary 2.6. Let $A=\langle a\rangle$ be cyclic of order $p^{s}$. Let $\chi_{p^{s}}$ be the character of the $\mathbb{Q} A$-module $V\left(p^{s}\right)$. Then $\chi_{p^{s}}$ is faithful and

$$
\chi_{p^{\prime}}\left(a^{i}\right)= \begin{cases}-p^{s-1}, & \text { if }\left(i, p^{s}\right)=p^{s-1}, \\ p^{s-1}(p-1), & \text { if } i=0, \\ 0, & \text { otherwise. }\end{cases}
$$

Proof. This follows from Lemma 2.5 .

If $n>1$ is a natural number and $n=p_{1}^{r_{1}} \ldots p_{t}^{r_{t}}$, where the $p_{i}$ are distinct primes, we define $n^{*}=\sum_{i=1}^{t} p_{i}^{r_{i}}$; define $1^{*}=0$. Note that, if $m \mid n$, then $m^{*} \leq n^{*}$. Thus, if $G \neq 1$ is a finite cyclic group, then $|G|^{*}=T(G)$.

Lemma 2.7. (1) Let $m$ be a positive integer. Then $2 \phi(m) \geq m^{*}$, unless $m=6$ when $2 \phi(m)=4$.

(2) Let $m=2^{\alpha} n$, where $\alpha \geq 0$ and $n$ is odd. Then $\frac{p}{p-1} \phi(m) \geq n^{*}$, for each prime divisor $p$ of $n$.

Proof. See [1, Lemma 3.5].

Let $G_{p}$ denote the Sylow $p$-subgroup of $G$. We define $\Omega_{1}\left(G_{p}\right)$ to be $\left\{z \in G_{p}: z^{p}=1\right\}$.

LEMma 2.8. Let $G$ be a finite abelian group, $p$ a prime and $K_{1}, \ldots, K_{s}$ subgroups of index $p$ in G. Let

$$
J=\left\{j: 1 \leq j \leq s, \bigcap_{i=1}^{j-1} K_{i} \cap \Omega_{1}\left(G_{p}\right) \neq K_{j}\right\} .
$$

Then for each $j \in J$ there is a subgroup $W_{j}$, cyclic of order $p$, such that $G$ is the direct product of the subgroups $W_{j}$ and the subgroup $\bigcap_{j \in J} K_{j}$.

Proof. We may assume that $J \neq \varnothing$. Our proof is by induction on $s$. If $s=1, J=\{1\}$ and $\bigcap_{i=1}^{0} K_{i}=G$, so that the hypothesis implies that there is an element $w$ of order $p$ in $K_{1}$. As $\left|G: K_{1}\right|=p, G$ is a direct product of $W=\langle w\rangle$ and $K_{1}$.

For $s>1$, let $J^{\prime}=J \cap\{1, \ldots, s-1\}$. By the induction hypothesis, for each $j \in J^{\prime}$ there is a subgroup $W_{j}$ of order $p$ such that $g$ is the direct product of the subgroups $W_{j}$ and the subgroup $H=\bigcap_{j \in J} K_{j}$. If $s \notin J$, we are done. So we assume that $s \in J$. It then suffices to show that $H$ is the direct product of a subgroup of order $p$ and the subgroup 
$\bigcap_{j \in J} K_{j}=K_{i} \cap H$. As $s \in J, \bigcap_{i=1}^{s-1} K_{j} \cap \Omega_{1}\left(G_{p}\right) \neq K_{s}$ so that $H \cap \Omega_{1}\left(G_{p}\right) \leq K_{s}$, and there is an element $w$ of order $p$ in $H$ but not in $K_{s}$. As $\left|G: K_{s}\right|=p$ we have $H K_{s}=G$. It follows that $\left|H: K_{s} \cap H\right|=\left|H K_{s}: K_{s}\right|=\left|G: K_{s}\right|=p$, so that $H$ is the direct product of $\langle w\rangle$ and $K_{s} \cap H$, as required.

Corollary 2.9. Let $G$ be a finite abelian group and $K_{1}, \ldots, K_{s}$ subgroups of index 6 in $G$. Let $J=\left\{j: 1 \leq j \leq s, \bigcap_{i=1}^{j-1} K_{i} \cap \Omega_{1}\left(G_{p}\right) \notin K_{j}\right.$ for $\left.p=2,3\right\}$. Let $n$ be maximal such that $G$ has a direct summand isomorphic to $C_{6}^{n}$. Then $n \geq|J|$.

Proof. Let $n_{p}$ be maximal such that $G$ has a direct summand isomorphic to $C_{p}^{n_{p}}$. By the Fundamental Theorem of Finitely Generated Abelian Groups, it suffices to show that $n_{2} \geq|J|$ and $n_{3} \geq|J|$ as $n=\min \left\{n_{2}, n_{3}\right\}$.

Let $p=2$. For each $j$, with $1 \leq j \leq s$, there is an element $x_{j}$ of order 3 not in $K_{j}$; put $K_{j}^{\prime}=K_{j}\left\langle x_{j}\right\rangle$, a subgroup of index 2 in $G$. It is clear that $K_{j}^{\prime} \cap \Omega_{1}\left(G_{2}\right)=K_{j} \cap \Omega_{1}\left(G_{2}\right)$. Thus we have

$$
\bigcap_{i=1}^{j-1} K_{1}^{\prime} \cap \Omega_{1}\left(G_{2}\right)=\bigcap_{i=1}^{j-1}\left(K_{i}^{\prime} \cap \Omega_{1}\left(G_{2}\right)\right)=\bigcap_{i=1}^{j-1}\left(K_{i} \cap \Omega_{1}\left(G_{2}\right)\right)=\bigcap_{i=1}^{j-1} K_{i} \cap \Omega_{1}\left(G_{2}\right) .
$$

If $j \in J$, then $\bigcap_{i=1}^{j-1} K_{i}^{\prime} \cap \Omega_{1}\left(G_{2}\right) \neq K_{j}$ and so

whence

$$
\bigcap_{i=1}^{j-1} K_{i}^{\prime} \cap \Omega_{1}\left(G_{2}\right) \neq K_{j} \cap \Omega_{1}\left(G_{2}\right)=K_{j}^{\prime} \cap \Omega_{1}\left(G_{2}\right),
$$

$$
\bigcap_{i=1}^{j-1} K_{i}^{\prime} \cap \Omega_{1}\left(G_{2}\right) \pm K_{j}^{\prime}
$$

The previous lemma implies that $G$ has a direct summand isomorphic to $C_{2}^{\mid J 1}$, so that $|J| \leq n_{2}$.

It follows similarly that $|J| \leq n_{3}$.

Lemma 2.10. Let $G \neq 1$ be a finite abelian group and let $n$ be maximal such that $G$ has a direct summand isomorphic to $C_{6}^{n}$. Also let $V$ be a $\mathbb{Q} G$-module. Suppose that $V$ is faithful for $G$, but no proper submodule of $V$ is faithful for $G$. Then $G$ contains an element $g$ such that $\chi_{v}(g)<0$ and

$$
\operatorname{dim} V-\chi_{v}(g) \geq T(G)-n
$$

Proof. Let $V=V_{1} \oplus \ldots \oplus V_{s}$, where each $V_{i}$ is an irreducible $\mathbb{Q} G$-module; let $K_{i}=C_{G}\left(V_{i}\right)$ and $K_{i}^{*}=\bigcap_{j \neq i} K_{j}$. Since $V$ is faithful, $\bigcap_{i=1}^{s} K_{i}=1$; also, as $V$ has no proper faithful submodule, $K_{i}^{*} \neq 1$ if $1 \leq i \leq s$. Let $K_{i, p}=K_{i} \cap G_{p}$. Choose a subset $I \subseteq\{1, \ldots, s\}$ minimal such that $\bigcap_{i \in I} K_{i, 2}=1$. Renumbering if necessary, we may assume that $I=$ $\{1, \ldots, t\}$ for some $t$. We interpret the case $t=0$ as corresponding to $G_{2}=1$.

Let $\left|G / K_{i}\right|=n_{i}$. Then $\operatorname{dim} V_{i}=\phi\left(n_{i}\right)$ since $V_{i}$ is the unique faithful module over $\mathbb{Q}$ for the cyclic group $G / K_{i}$; namely, $V_{i}$ is isomorphic to $\mathbb{Q}(\omega)$, where $\omega$ is a primitive $n_{i}$-th root of unity and the generator of $G / K_{i}$ acts as multiplication by $\omega$. 
For each $j$, where $1 \leq j \leq t$, let $x_{j}$ be an involution in $\bigcap_{\substack{i=1 \\ i \neq j}}^{t} K_{i, 2}$, and $x=x_{1} \ldots x_{i}$. Then $x$ is an involution and acts as an involution on each of $V_{1}, \ldots, V_{i}$; therefore, it acts as -1 on each of these modules. [See Note (3) in Chapter 1.] Now renumber the $V_{i}$ so that $V_{1}, \ldots, V_{u}$ are precisely those on which $x$ acts as -1 . Then $x$ acts trivially on $V_{u+1}, \ldots, V_{s}$. For $j=u+1, \ldots, s$, choose $x_{j}$ of prime order in $K_{j}^{*}$, and let $g=x x_{u+1} \ldots x_{s}=$ $x_{1} \ldots x_{r} x_{u+1} \ldots x_{s}$. Thus, $g$ acts as -1 on each of $V_{1}, \ldots, V_{u}$ and as an element of order $p_{j}$ on $V_{j}$ if $u+1 \leq j \leq s$. By Lemma 2.5 we have $\chi_{v_{j}}(g)=-\operatorname{dim} V_{j}$ if $1 \leq j \leq u$, and $\chi_{v}(g)=-\frac{1}{p_{j}-1} \operatorname{dim} V_{j}$ if $u+1 \leq j \leq s$. Hence we have $\chi_{v}(g)<0$ and

$$
\operatorname{dim} V-\chi_{v}(g)=2 \sum_{j=1}^{u} \operatorname{dim} V_{j}+\sum_{j=u+1}^{s}\left(1+\frac{1}{p_{j}-1}\right) \operatorname{dim} V_{j}
$$

For $0 \leq j \leq s$, define $I_{j}=\bigcap_{i=1} K_{i}$, so that $I_{0}=G$. Let

$J_{0}=\{j: u+1 \leq j \leq s\}$,

$J_{1}=\left\{j: 1 \leq j \leq u,\left|G: K_{j}\right|=6, I_{j-1} \cap \Omega_{1}\left(G_{p}\right) \neq K_{j}\right.$ for $\left.p=2,3\right\}$,

$J_{2}=\left\{j: 1 \leq j \leq u,\left|G: K_{j}\right|=6, I_{j-1} \cap \Omega_{1}\left(G_{2}\right) \leq K_{j}\right\}$,

$J_{3}=\left\{j: 1 \leq j \leq U,\left|G: K_{j}\right|=6, I_{j-1} \cap \Omega_{1}\left(G_{3}\right) \leq K_{j}, i_{j-1} \cap \Omega_{1}\left(G_{2}\right) \not K_{j}\right\}$,

and $J_{4}=\left\{j: 1 \leq j \geq u,\left|G: K_{j}\right| \neq 6\right\}$.

Define subgroups $M_{j}$ of $G$ as follows:

$$
M_{j}= \begin{cases}K_{j}, & \text { if } j \in J_{1} \cup J_{4} \\ G_{2} K_{j}, & \text { if } j \in J_{0} \cup J_{2} \\ G_{3} K_{j}, & \text { if } j \in J_{3}\end{cases}
$$

Let $m_{j}=\left|G: M_{j}\right|$ so that:

(a) for $j \in J_{0}, m_{j}$ is the maximal odd divisor of $n_{j}$ and so, by Lemma 2.7(2), $\frac{p_{j}}{p_{j}-1} \operatorname{dim} V_{j} \geq m_{j}^{*}$

(b) for $j \in J_{1}, m_{j}=n_{j}=6$ so that $\operatorname{dim} V_{j}=\phi(6)=2$ while $m_{j}^{*}=5$;

(c) for $j \in J_{2}, m_{j}=3, n_{j}=6$ so that $\operatorname{dim} V_{j}=2$ while $m_{j}^{*}=3$;

(d) for $j \in J_{3}, m_{j}=2, n_{j}=6$ so that $\operatorname{dim} V_{j}=2$ while $m_{j}^{*}=2$; $m_{j}^{*}$.

(e) for $j \in J_{4}, m_{j}=n_{j} \neq 6$ so that $\operatorname{dim} V_{j}=\phi\left(n_{j}\right)$ and so, by Lemma $2.7(1), 2 \operatorname{dim} V_{j} \geq$

It follows that

$$
2 \sum_{j=1}^{u} \operatorname{dim} V_{j}+\sum_{j=u+1}^{s}\left(1+\frac{1}{p_{j}-1}\right) \operatorname{dim} V_{j} \geq \sum_{j \in J_{1}} m_{j}^{*}-\left|J_{1}\right|+\sum_{j \in J_{1}} m_{j}^{*} .
$$

It follows from Corollary 2.9 that $n \geq\left|J_{1}\right|$, so that we have

$$
\operatorname{dim} V-\chi_{V}(g) \geq \sum_{j=1}^{s} m_{j}^{*}-n
$$

We next show that $\bigcap_{j=1}^{s} M_{j}=1$. Suppose that this is not the case and that $m$ is an 
element of prime order $p$ in this intersection. As $\bigcap_{j=1}^{s} K_{j}=1$, there is a minimal index $j$ for
which $m \notin K_{j}$.

Suppose that $p=2$. Then $j \notin J_{1} \cup J_{4}$ as here $M_{j}=K_{j}$. Also $j \notin J_{3}$ as here $M_{j}=G_{3} K_{j}$ and so $M_{j} / K_{j} \cong G_{3} / G_{3} K_{j}$, a 3-group. If $j \in J_{0}$, then $m \in K_{i}$, for $1 \leq i \leq t$, so that $m \in I_{t} \cap G_{2}$ which is trivial; this is a contradiction. If $j \in J_{2}$, then by the minimality of $j$, $m \in I_{j-1} \cap \Omega_{1}\left(G_{2}\right)$ so that $m \in K_{j}$, by the definition of $J_{2}$, again a contradiction.

Suppose that $p=3$. As before $j \notin J_{0} \cup J_{1} \cup J_{2} \cup J_{4}$. If $j \in J_{3}$, then $m \in I_{j-1} \cap \Omega_{1}\left(G_{3}\right) \leq$ $K_{j}$, a contradiction.

The case $o(m) \geq 5$ also leads to a contradiction as the Sylow $p$-subgroup of $M_{j}$ is contained in $K_{j}$ for each $p \neq 2,3$ and for all $j, 1 \leq j \leq s$.

As $\bigcap_{j=1}^{s} M_{j}=1, G$ can be embedded as a subgroup of the direct product $\operatorname{Dr}_{j=1}^{s} G / M_{j}$. However from [2],

$$
T(G) \leq T\left(\mathrm{Dr}_{j=1}^{s} G / M_{j}\right)=\sum_{j=1}^{s} T\left(G / M_{j}\right)=\sum_{j=1}^{s} m_{j}^{*}
$$

From (2), we deduce the inequality

$$
\operatorname{dim} V-\chi_{v}(g) \geq T(G)-n
$$

as required.

THEOREM 2.11. Let $G \neq 1$ be a finite abelian group and let $n$ be maximal such that $G$ has a direct summand isomorphic to $C_{6}^{n}$. Then

$$
c(G)=q(G)=T(G)-n .
$$

Proof. By Corollary 2.2 we have $c(G)=q(G)$.

Now let $V$ be a faithful quasi-permutation representation of $G$ over $\mathbb{Q}$ of minimal degree. Then $q(G)=\operatorname{dim} V$. Write $V=V_{1} \oplus W$, where $V_{1}$ is a faithful $\mathbb{Q} G$-module with no proper faithful submodules for $G$. By Lemma 2.10, there is $g \in G$ such that

$$
\operatorname{dim} V_{1}-\chi_{v_{1}}(g) \geq T(G)-n
$$

and $\chi_{v_{1}}(g)<0$. Since $\chi_{v}(g) \geq 0$ we have

$$
\begin{aligned}
0 \leq \chi_{v}(g)= & \chi_{v_{1}}(g)+\chi_{w}(g) \leq \operatorname{dim} V_{1}-(T(G)-n)+\chi_{w}(g) \\
& \leq \operatorname{dim} V_{1}-(T(G)-n)+\operatorname{dim} W
\end{aligned}
$$

Hence

$$
\operatorname{dim} V=q(G) \geq T(G)-n
$$

Now we show that there exists a quasi-permutation module $U$ over $\mathbb{Q}$ for $G$ such that $\operatorname{dim} U=T(G)-n$ and, since $T(G)-n$ is the minimal value, we have $q(G)=T(G)-n$.

Let $G=\prod_{i=1}^{s} G_{i}$. Here $G_{i} \cong C_{6}$, where $p_{i}$ is a prime, $m_{i}$ is a positive integer for $i=1, \ldots, n$, and $G_{i} \cong C_{p_{i}^{m}}$ for $i=n+1, \ldots, s$. Let $K_{i}=\prod_{j \neq i} G_{j}$ for $i=1, \ldots, s$. Then 
$\bigcap_{i=1}^{s} K_{i}=1$ and $G / K_{i}$ is cyclic. Let $\chi_{i}$ be the faithful irreducible $\mathbb{Q}$-character of $G / K_{i} \cong G_{i}$; (see Lemma 2.5). Let $V_{i}$ be its module and let $U_{1}=\bigoplus_{i=1}^{s} V_{i}$. Then, by Lemma 2.5 and Corollary 2.6, we have:

$\operatorname{dim} V_{i}=2$ for $i=1, \ldots, n$, and $\min \left\{\chi_{i}(g): g \in G\right\}=-2$ for $i=1, \ldots, n$;

$\operatorname{dim} V_{i}=p_{i}^{m_{i}-1}\left(p_{i}-1\right)$ for $i=n+1, \ldots, s, \quad$ and $\min \left\{\chi_{i}(g): g \in G\right\}=-p_{i}^{m_{i}-1}$

for $i=n+1, \ldots, s$. Hence $\operatorname{dim} U_{1}=\sum_{i=1}^{s} \operatorname{dim} V_{i}$ and

$$
A=\min \left\{\chi_{U_{1}}(g): g \in G\right\} \geq \sum_{i=1}^{s} \min \left\{\chi_{i}(g): g \in G\right\} .
$$

Let $l=-A$ and let $l \mathbb{Q}$ denote the direct sum of $l$ copies of the trivial module. Let $U=U_{1}+l \mathbb{Q}$, so that $U$ is a faithful quasi-permutation module over $\mathbb{Q}$. Hence by (4) we have $T(G)-n \leq q(G)$ and, by the definition of $q(G)$, we have $q(G) \leq \operatorname{dim} U$. It follows that

$$
T(G)-n \leq \operatorname{dim} U=\operatorname{dim}\left(U_{1} \oplus l \mathbb{Q}\right) \leq \operatorname{dim} U_{1}-\sum_{i=1}^{s} \min \left\{\chi_{i}(g): g \in G\right\}=T(G)-n
$$

Hence $\operatorname{dim} U=T(G)-n$, as required.

Acknowledgement. This paper is a part of a Ph.D thesis submitted to the University of Manchester. The work was done under the supervision of Professor Brian Hartley (1992-94). Also I would like to express my sincere gratitude to Dr. Robert Sandling for help in preparing this paper.

\section{REFERENCES}

1. J. M. Burns, B. Goldsmith, B. Hartley and R. Sandling, On quasi-permutation representations of finite groups, Glasgow Math. J. 36 (1994), 301-308.

2. M. Hoffman, An invariant of finite abelian groups. Amer. Math. Monthly 94 (1987), 664-666.

3. I. M. Isaacs, Character theory of finite groups (Academic Press, New York, 1976).

4. S. K. Sehgal, Topics in group rings (Marcel Dekker, New York, 1978).

5. W. J. Wong, Linear groups analogous to permutation groups, J. Austral. Math. Soc. Ser. A 3 (1963), 180-184.

6. W. J. Wong, On linear p-groups, J. Austral. Math. Soc. Ser. A 4 (1964), 174-178.

Department of Mathematics

UNIVERSITY of MANCHESTER

MANCHESTER M13 9PL

ENGLAND

Present address:

Department of Mathematics

UNIVERSITY OF URMIA

URMIA

IRAN 